\title{
Study on Image Recognition and Classification of Wood Skin Defects Based on BOW Model
}

\author{
Fan Yang ${ }^{1, ~ a, ~ Y u z e n g ~ W a n g ~} 2$, b \\ ${ }^{1}$ School of Mechanical Engineering, University of Jinan, Jinan 250022, China; \\ ${ }^{2}$ School of Mechanical Engineering, University of Jinan, Jinan 250022, China. \\ ayangfchn@foxmail.com, bme_wangyz@ujn.edu.cn
}

Keywords: wood skin defect, image recognition, feature extraction, BOW model, support vector machine.

\begin{abstract}
To solve the problem of wood skin recognition and classification in wood processing industry, a method based on BOW model for image recognition of wood skin defects is proposed. First, we extract the HOG feature of wood skin defect image, and then build the BOW model to describe the wood skin defect image. Finally, we use different kernel functions combined with support vector machine (SVM) to identify the types of wood skin defects. The experimental results show that the average recognition rate of the proposed method is $85.4 \%$ for wood skin defect image recognition and classification, indicating that the BOW model is effective and feasible for wood skin defect image recognition and classification.
\end{abstract}

\section{Introduction}

The wood-based panel industry is one of the pillar industries of forestry efficient utilization of wood and timber resources and is an important part of the sustainable development strategy of forestry. Among the wood-based panel products in China, plywood started first and has the fastest development speed. Plywood has always been the leading product in the wood-based panel industry in China. It is mainly used in furniture manufacturing, decoration, packaging and building forms [1]. With the improvement of people's material living level, many man-made plywood and its products are needed to meet the huge demand of furniture, decoration and construction industry. The quality of veneer as primary material of plywood is particularly important. In 2016, the output of wood plywood in China reached 1.6382 trillion cubic meters, and the demand for veneer raw materials was very large. However, due to the defects in veneer surface, the veneer was not fully utilized. Therefore, the classification of veneer defects was investigated. To improve the overall utilization of veneer and reduce the cost of raw materials, the research results will bring huge economic benefits to the wood processing industry, with important theoretical significance and broad application prospects.

Currently, there are two commonly used methods for intelligently identifying and classifying wood images. One is the analysis of unique images based on the unique textures and colors of the wood surface. These methods generally treat an image as an independent image. Individuals are therefore susceptible to features such as light intensity, rotation angle, and scaling that appear on individual images. This method is suitable for the taxonomy of fewer tree species. Another way is to get information in digital image by image processing technology. For wood defect detection, the image information can be a defect type [2]. Due to the low dependence and high classification efficiency of wood surface defect detection methods, computers and simple image acquisition devices can be implemented. In recent years, with the rapid development of image processing technology and computer, the Bag of Words algorithm has been widely applied. In this paper, the Bag of Words algorithm and its application in the classification and recognition of wood skin have been deeply studied. 


\section{Construction of BOW Model Based on Wood Skin Defect Image}

The Bag of Words model is originally used in the field of text processing, which is used to identify and classify text documents. The Bag of Words model introduces automatic document classification technology to image recognition. The main idea is to regard an image as a large number of visual words. Each visual word is quantized local feature descriptor, and each local feature can form a characteristic word. Such an image can be expressed as a combination of multiple feature words. The number of each characteristic word appearing in the image potentially indicates the category of the image. The method in object recognition testing more classes have won good recognition results, using it to do image visual word bag model, need to map the two-dimensional image information into visual keyword set, such already save the image of local characteristics and effectively compress the image description [3-4]. The process of constructing BOW for the image of the wood defect is shown in Fig. 1.

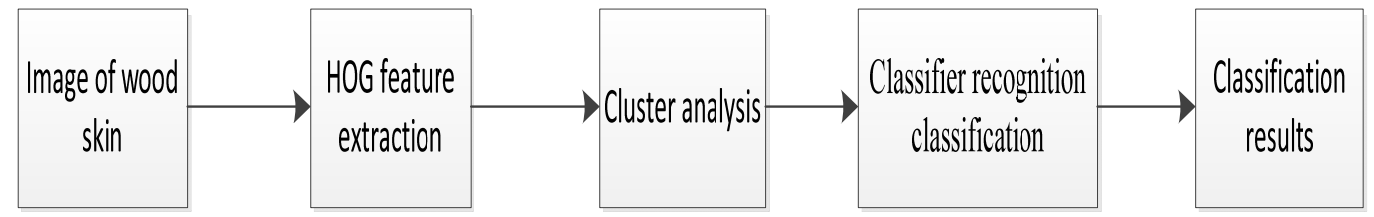

Fig 1. The process of constructing BOW for the image

\subsection{Section Headings.}

Histogram of Oriented Gradient (HOG) is a feature descriptor for object detection in computer vision and image processing. It consists of the gradient direction histogram of the local region of the image, which can maintain a good invariance for both the image geometry and the optical deformation. In this paper, HOG descriptors are used to extract the characteristics of the skin defects. The following are four steps for HOG feature extraction:

1) Image preprocessing normalization. The color image of the collected wood skin is gray, and the Gamma correction method is used to normalize the color space of the input image.

2) Gradient calculation. The gradient of the vertical and horizontal direction of the image of the wood skin after gray scale is calculated respectively.

3) Statistical cell histogram. The skin image is divided into a number of $10 \times 10$ pixel cell units (cell), and the histogram of 9 bin is used to calculate the gradient information of the $10 \times 10$ pixels. The gradient direction of cell is divided into 360 direction blocks, and each pixel in cell is weighted projected by histogram in gradient direction. The histogram of gradient direction of cell is obtained.

4) Statistical block histogram. Each cell unit is combined into a connected interval (blocks), and a feature vector of all cell in a block is connected to the HOG feature of the block. The interval is overlapped. This means that every cell's characteristics will appear in the last feature vector many times with different results. The normalized block descriptor (vector) is called HOG descriptor.

5) Collect the HOG features. Combine all the histogram vectors in the block into a large HOG eigenvector.

\subsection{Cluster Analysis.}

After the feature extraction, the visual defects of the veneer defects represented by the local feature descriptors are generated. The visual words are clustered into K categories, and the cluster centers of each category are used as the descriptions of the corresponding visual words. The K-means mean clustering method is used to cluster the veneer defect images represented by the feature descriptors to obtain multiple clusters. In the clustering center, each clustering center is a visual word. All the centers are clustered to form the visual dictionary of the BOW model [5-6].

$\mathrm{N}$ data sets $\{\mathrm{X} 1, \mathrm{X} 2, \ldots \mathrm{Xn}\}$ is divided into $\mathrm{k}$ classes $\{\mathrm{C} 1, \mathrm{C} 2, \ldots \mathrm{Ck}\}$, the same category has high similarity, the similarity of different categories is relatively low, the K-means algorithm [7] procedures are as follows:

(1) Randomly select $\mathrm{k}$ from the given dataset sample as the initial cluster center.

(2) Using the principle of nearest neighbors, the distance between each data sample point and the cluster must first be calculated, and then the distance to each data sample is allocated to the nearest cluster based on the distance. 
(3) Through the calculation, the average calculated all the data values to determine each new data center with the average value.

(4) To judge whether the clustering function (1-1) is satisfied or not, if the algorithm is satisfied, the algorithm can be finished, and the corresponding clustering results can be obtained. Instead, jump to step 2 .

$$
\mathrm{J}=\sum_{j=1}^{n} \sum_{i=1}^{n}\left\|x_{i}^{(j)}-c_{j}\right\|^{2}
$$

After obtaining the visual dictionary, the content of every wooden skin defect image can be re expressed by the form of visual histogram, which is the table method of visual characteristics in BOW model. The purpose of this expression is to classify and identify the images with the classifier, and solve the problem that the HOG features can not be classified directly by the classifier.

\subsection{Classifier Recognition Classification.}

\subsubsection{Selection Classifier.}

Currently in the field of image classification, commonly used classifiers are Adaboost classifier, support vector machine (SVM), and random forest classifier (RF). SVM is a classification algorithm based on nonlinear prediction of turbidity theory, which can effectively avoid Fitting, poor generalization and other shortcomings. Because of its versatility, robustness, simple calculation, and good results, it has been widely used in robot vision, pattern recognition, regression prediction and other fields of application and research [8], this article's experiment is also under the SVM classifier ongoing. To facilitate the classification, the SVM classifier is firstly trained by using the BOW model description of the veneer defect image, and then the trained SVM classifier is used to classify the test sample to obtain the recognition results of different defect images.

\subsubsection{Select the Kernel Function}

Kernel function is a very important parameter in SVM model. When support vector machine is used for pattern recognition and classification, it is necessary to select a suitable kernel function, but how to select a reasonable kernel function in practical application is a difficult problem [9]. Therefore, for the problem of classification and identification of veneer defects, the commonly used Gaussian Kernel (Gaus), Polynomial Kernel (Poly), Chi-Square Kernel (Chi), and Histogram intersection kernel function (Hist) are used in this experiment. The four kernel functions classify and analyze the veneer defect images, and simultaneously test the PCA dimension (the number of words) to determine the optimal PCA dimension. This experiment selects the dimension 32, 64, 128 for test analysis.

\section{Experimental Results and Analysis}

Based on the BOW model and the SVM classifier, this paper makes a classification simulation experiment on the wood skin defect image. The hardware platform of the experiment is: CPU, Intel Core i7-7700 (four core, 3.60GHz); memory, 8G. Choose from the common defects of veneer defect image sample database of 4 kinds of veneer are hole, dead section, movable joint and crack, each type of veneer defect image, select 90 pieces of veneer defect image as the training sample, selected 60 corresponding veneer defect images as a test sample, the image size is $200 \times 200$. The software uses Microsoft Visual Studio.

Table 1.The results of four classes of kernel functions for the identification of wood defects (dimension: 32)

\begin{tabular}{cccccc}
\hline \multirow{2}{*}{ Kernel function } & Dead knot & Slipknot & Crack & Hole & Average recognition rate \\
\hline Gaus & $71.7 \%$ & $81.7 \%$ & $80.0 \%$ & $86.7 \%$ & $80.0 \%$ \\
Poly & $88.3 \%$ & $85.0 \%$ & $76.7 \%$ & $78.3 \%$ & $82.1 \%$ \\
Chi & $83.3 \%$ & $85.0 \%$ & $80.0 \%$ & $81.7 \%$ & $82.5 \%$ \\
Hist & $80.0 \%$ & $83.3 \%$ & $83.3 \%$ & $78.3 \%$ & $81.2 \%$ \\
\hline
\end{tabular}


Table 2. The results of four classes of kernel functions for the identification of wood defects (dimension: 64)

\begin{tabular}{cccccc}
\hline \multirow{2}{*}{ Kernel function } & Dead knot & Slipknot & Crack & Hole & Average recognition rate \\
\hline Gaus & $81.7 \%$ & $83.3 \%$ & $83.3 \%$ & $86.7 \%$ & $83.8 \%$ \\
Poly & $83.3 \%$ & $85.0 \%$ & $81.7 \%$ & $85.0 \%$ & $83.8 \%$ \\
Chi & $83.3 \%$ & $86.7 \%$ & $85.0 \%$ & $83.3 \%$ & $84.6 \%$ \\
Hist & $81.7 \%$ & $85.0 \%$ & $86.7 \%$ & $83.3 \%$ & $84.2 \%$ \\
\hline
\end{tabular}

Table 3.The results of four classes of kernel functions for the identification of wood defects (dimension: 128)

\begin{tabular}{cccccc}
\hline \multirow{2}{*}{ Kernel function } & Dead knot & Slipknot & Crack & Hole & Average recognition rate \\
\hline Gaus & $81.7 \%$ & $83.3 \%$ & $83.3 \%$ & $88.3 \%$ & $84.2 \%$ \\
Poly & $88.3 \%$ & $85.0 \%$ & $78.3 \%$ & $83.3 \%$ & $83.7 \%$ \\
Chi & $88.3 \%$ & $86.7 \%$ & $86.7 \%$ & $80.0 \%$ & $85.4 \%$ \\
Hist & $88.3 \%$ & $86.7 \%$ & $83.3 \%$ & $80.0 \%$ & $84.6 \%$ \\
\hline
\end{tabular}

From the data in Table 1 to Table 3, it can be seen that there are differences in the recognition results of the four types of veneer defect images by the four types of kernel functions, of which the Chi kernel function has the best recognition effect on the four kinds of veneer defects images, and at the same time, the experimental results also shows that with the increase of the optimal dimension of the kernel function, the average recognition rate of the detection and recognition of the four types of kernel functions shows an overall increasing trend.

Table 4. Recognition time results of four classes of kernel functions with different dimensions

\begin{tabular}{cccccc}
\hline Dimensions & Gaus & Poly & Chi & Hist & Average time $/ \mathrm{s}$ \\
\hline 32 & 2.048 & 1.993 & 2.021 & 2.050 & 2.028 \\
64 & 2.159 & 2.059 & 2.186 & 2.126 & 2.133 \\
128 & 2.783 & 2.787 & 2.819 & 2.769 & 2.790 \\
\hline
\end{tabular}

Through the experimental data of Table 4 , it is shown that with the increase of the experimental dimension, the recognition time of the four types of kernel functions for the defect of the wood is also increasing.

\section{Summary}

In this paper, we build a BOW bag model based on HOG features. The model divides the classified image into several sub regions, and extracts corresponding features to form visual words, to build a spatial visual dictionary for training wood skin defect images. At the same time, we use different kernel functions to simulate wood skin defect images. The method has verified the best applicable kernel function and achieved a good recognition rate, which is of great significance for the practical application of recognition system.

At the same time, by analyzing the above experimental results, how to improve the recognition efficiency of kernel function based on guaranteeing the recognition rate of kernel function will be the focus of further research. To solve this problem, we need further exploration through experiments.

\section{References}

[1]. Wenjun Sun, Baodong Cheng, Xinsheng Pang. Chinese plywood international competitiveness comparative analysis [J]. Journal of Beijing Forestry University (SOCIAL SCIENCE EDITION). Vol. 13 (2014) No. 4, p. 67-68.

[2]. Yuehui Lu, Xiaorong Xu. Research on wood recognition based on Bag of Words [J]. Modern computer (Professional Edition). Vol. 2 (2012) No. 3, p.19-21. 
[3]. Xufeng Zhu, Caiwen Ma, Paul Liu. Automatic classification of air targets using improved word bag model [J]. Infrared and laser engineering. Vol. 41 (2012) No. 5, p. 1385-1386.

[4]. Yuxin Wang, He Guo, Changqin He, Zhen Feng, Qi Jia. Space visual word bag model for image scene classification [J]. Computer science. Vol. 38 (2011) No. 8, p. 265-266.

[5]. Linbo Xie: Research on the classification of image model of oil tea pests based on SVM (master, Central South University of Forestry and Technology, China 2015). p.36-38.

[6]. Beiji Zou, Jianjing Guo, Chengzhang Zhu, Wenjun Yang, Hui Wu, Qi He. BOW-HOG feature image classification of [J]. Vol. 51 (2017) No. 12, p. 2-3.

[7]. Zhe Zhang, Junxi Zhang, Huifeng Xue. Improved K-means Clustering Algorithm [J]. In Conference and Signal Processing. Vol. 5 (2008) No. 7, p. 169-172.

[8]. Burges C.J.C. Atutorial on support vector machines for Pattern recognition[J]. Data Mining and Knowledge Discovery, Vol. 2 (1998) No. 2, p. 121-126.

[9]. astanlar Y, Temizel A, Yardimci Y. Improved SIFT matching for image pairs with scale difference[J]. Electronics Letters, Vol. 46 (2010) No. 5, p. 107-108. 